\title{
Enhanced Trellis Coded Multiple Access (ETCMA)
}

\author{
Alberto G. Perotti and Branislav M. Popović \\ Huawei Technologies Sweden AB \\ Skalholtsgatan 9-11, SE-16494 Kista, Sweden \\ E-mails: \{alberto.perotti, branislav.popovic\}@huawei.com
}

\begin{abstract}
We propose an enhanced version of trellis coded multiple access (TCMA), an overloaded multiple access scheme that outperforms the original TCMA in terms of achieved spectral efficiency. Enhanced TCMA (ETCMA) performs simultaneous transmission of multiple data streams intended for users experiencing similar signal-to-noise ratios and can be employed both in the uplink and in the downlink of wireless systems, thus overcoming one of the main limitations of TCMA. Thanks to a new receiver algorithm, ETCMA is capable of delivering a significantly higher spectral efficiency. We show that ETCMA approaches the capacity of the Additive White Gaussian Noise channel for a wide range of signal-to-noise ratios.
\end{abstract}

\section{INTRODUCTION}

Next generation wireless systems will have to face the demand for higher aggregate data rates while being capable of providing reliable communication to many simultaneous users and applications. They will not necessarily be able to deliver higher per-user data rates, but higher aggregate data rates to a growing number of users. To this purpose, novel efficient Multiple Access (MA) techniques are required. Combined with high-performance coding and modulation schemes, these techniques will be able to achieve higher aggregate and per-user Spectral Efficiencies (SE) by means of a more efficient use of the channel's physical resources. Conventional MA techniques typically perform orthogonal multiplexing of several coded and modulated data streams intended for different users. Each stream is transmitted using a different set of physical timefrequency-space Resource Elements (REs), i.e. without using REs for simultaneous transmissions of multiple streams.

Overloading is a paradigm according to which, in a multiuser transmission system, the number of users is greater than the dimension of the signal space [1]. In the context of CodeDivision Multiple Access (CDMA), overloading means using a number of spreading sequences larger than the length of each sequence. This concept has been recently extended beyond the context of CDMA and new OverLoaded Multiple Access (OLMA) schemes have been devised. In OLMA schemes, several data streams are multiplexed and transmitted using the same REs in order to provide increased data rates by achieving significantly higher spectral efficiency than conventional MA schemes.

In our scenario, the optimization target is the maximization of the aggregate Down-Link (DL) spectral efficiency (of one transmitter) by simultaneous transmission to user equipment (UE) devices experiencing similar physical channel qualities. UEs that report to the DL transmitter the same channel quality indicator value are grouped by the scheduler into the same category, and then served by the same set of REs. The corresponding OLMA methods preserve the same data rate, the same transmitted energy per bit of each multiplexed stream, and the same scheduler design as if each of the multiplexed streams would have been transmitted alone on the observed timefrequency-space resources. It further means that the transmitted power per RE is increased proportionally to the overloading factor, i.e. the number of multiplexed streams. OLMA schemes designed using this principle include, for example, Low-Density Spread (LDS) MA (LDSMA) [2]-[4], Interleave-Division MA (IDMA) [5] and Trellis-Coded MA (TCMA) [6], [7].

All the aforementioned schemes perform stream multiplexing by superposition of coded and modulated signals by adopting different solutions. IDMA adopts a Bit-interleaved Coded Modulation (BICM) [8] approach with very low-rate codes. LDSMA schemes use low-density signatures to enable detection by low-complexity receivers. The aggregate SE of these schemes approaches the Additive White Gaussian Noise (AWGN) channel capacity in the low Signal-to-Noise Ratio (SNR) region, where the maximum SE is limited to few bits/s/Hz. In the high-SNR region their SE diverges from the AWGN channel capacity, thus requiring a significant increase of transmitted power in order to reach high aggregate spectral efficiency.

We fill this gap by proposing a new MA scheme based on TCMA that, thanks to a carefully designed transmission scheme and to an improved receiver algorithm, exhibits a performance close to the AWGN channel capacity for a wide range of SNR values and features aggregate $\mathrm{SE}$ values as high as $7 \mathrm{bits} / \mathrm{s} / \mathrm{Hz}$. Moreover, the new OLMA scheme can be used both in the downlink and in the uplink of wireless systems.

This paper is organized as follows: Sec. II describes the ETCMA transmitter and receiver schemes, Sec. III presents the obtained results and Sec. IV draws the final conclusions.

\section{THE ETCMA SCHEME}

TCM has been proposed in [9] as a coded-modulation scheme in which the modulation is an integral part of the encoding process. It has been shown that it is possible to achieve significant coding gains even using simple convolutional codes with a low number of states, achieving improved performance w.r.t. BICM [8] when transmitting on the AWGN channel.

In a TCMA system, each UE transmits a single TCMencoded and modulated data stream in the same channel 


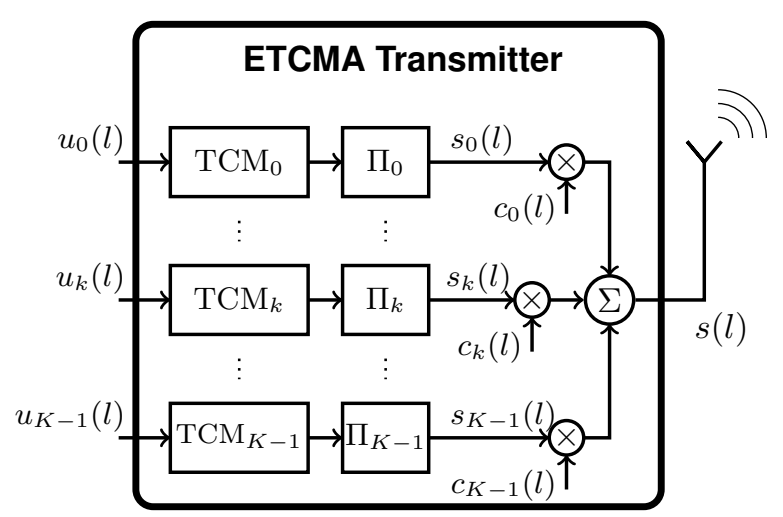

(a) Transmitter.

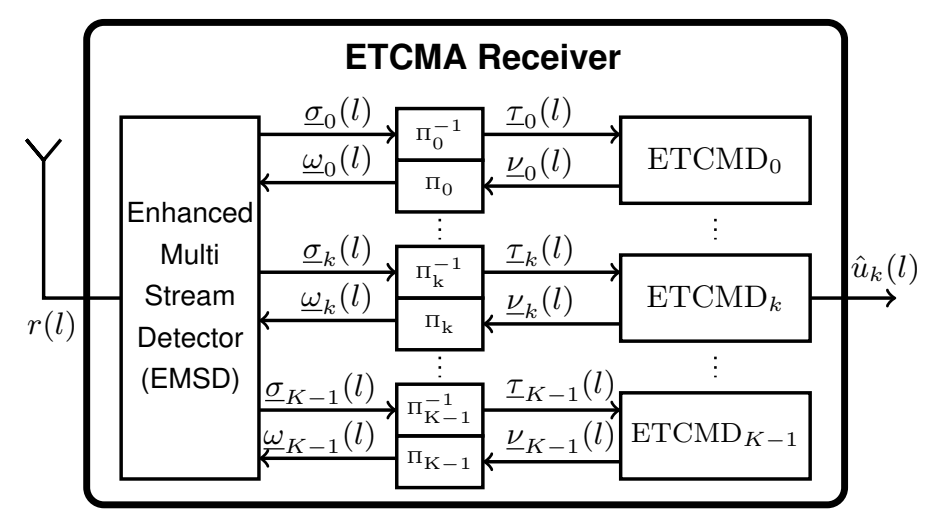

(b) Receiver. Here, $\underline{\sigma}_{k}(l)$ is a shorthand notation for vector $\left\{\sigma_{k, n}^{(t)}(l)\right\}_{n=0}^{\left|\chi_{\mathrm{TCM}}\right|-1}$. A similar notation is used for $\tau, \nu$ and $\omega$.

Fig. 1. ETCMA transmission system.

resources already used by other UEs. As a result, the received signal is the superposition of all the transmitted signals. In ETCMA, superposition of streams is performed at the transmitter by a suitably designed linear combiner whose structure is detailed hereinafter.

\section{A. ETCMA Transmitter}

The ETCMA transmitter (see Fig. 1(a) performs concurrent transmission of multiple independent data streams, possibly intended for different users, by a single transmitter. Here, $K$ data streams consisting of information words of size $L$ bits are independently encoded and modulated using TCM encoders $\mathrm{TCM}_{k}$ then interleaved by a stream-specific permutation $\Pi_{k}$. Each interleaved stream is multiplied by a specific scrambling sequence $c_{k}(l)$, then multiple streams are summed and transmitted. Stream scrambling and summing are the new features of the ETCMA scheme w.r.t. to original TCMA.

Each stream may be encoded using different trellis codes or modulation schemes, although we assume hereinafter that all streams use the same TCM scheme whose parameters are chosen as a function of $K$ using a criterion described in Sec. III-C.

Modulated streams are interleaved using permutations $\Pi_{k}$ which have to be stream-specific in order to help the receiver to separate signals. The set of permutations can be generated choosing $K$ distinct permutations of the same size $L$ with uniform distribution from the set of permutations of a certain size. As an alternative, the permutation set can be obtained by circular shifts of a single randomly generated permutation. As a further alternative, Quadratic Polynomial Permutations [10] can be used. In all cases, we observed a similar performance. Hereinafter, we adopt random permutations.

Scrambling signatures are used to further separate streams in the signal domain so that their discrimination is facilitated. After interleaving, streams are scrambled and combined before transmission. Scrambling consists in multiplying the sequence of interleaved symbols $s_{k}(l)$ by a stream-specific signature of complex coefficients $\mathbf{c}_{k}=\left(c_{k}(l)\right)$,where $l=0, \ldots, L-1$ is the symbol index. The transmitted signal is therefore

$$
s(l)=\sum_{k=0}^{K-1} c_{k}(l) s_{k}(l)
$$

and belongs to a set of symbols $\chi_{\mathrm{SUP}}(l)=\left\{x_{q}(l)\right\}$ called super-constellation ${ }^{1}[11]$ whose size is $\left|\chi_{\mathrm{TCM}}\right|^{K}$. Here, $\chi_{\mathrm{TCM}}$ is the TCM constellation and $x_{q}(l), q=0, \ldots,\left|\chi_{\mathrm{TCM}}\right|^{K}-1$, is the $(q+1)$-th symbol of super constellation $\chi_{\mathrm{SUP}}(l)$.

For the design of scrambling signatures, different approaches have been undertaken. The first approach consists in choosing the scrambling coefficients that maximize the minimum Euclidean distance $d_{E, \min }\left(\chi_{\mathrm{SUP}}(l)\right)$ between symbols of the super-constellation. In this case, since $\chi_{\mathrm{TCM}}$ is not timevarying, the obtained signature coefficients are independent of time index $l$. Their values have been found by numerical optimization. According to a second option, we choose the coefficients as uniformly spaced in a given interval $[0, \pi / \mu)$, where $\mu$ is a parameter whose value depends on the shape of $\chi_{\mathrm{TCM}}$. The upper interval endpoint $\pi / \mu$ is the smallest phase rotation that maps $\chi_{\mathrm{TCM}}$ into itself. We have $\mu=1$ for BPSK, $\mu=2$ for QPSK and QAM constellations, $\mu=4$ for 8PSK, etc. In this case, the scrambling coefficients are defined as

$$
c_{k}(l)=\exp (j \pi k /(K \mu)), \quad k=0, \ldots, K-1 .
$$

A third option consists in using a set of signatures selected from the Zadoff-Chu (ZC) class [12], a type of complex sequences with low cross-correlation properties:

$$
\left.c_{k}(l)=\exp \left(-\left(j \pi r_{k} l(l+L \bmod 2)\right) / L\right)\right), l=0, \ldots, L-1
$$

where $\left\{r_{k}\right\}$ is a set of distinct stream-specific integers relatively prime with $L$.

For each value of $K$, the receiver performance is evaluated by using each of the above three sequence designs. The design resulting in the best performance is selected for inclusion in the set of transmission parameters shown in Tab. []

\footnotetext{
${ }^{1}$ Please note that, although $\chi_{\mathrm{TCM}}$ does not depend on $l, \chi_{\mathrm{SUP}}$ may be time-varying because of the presence of different scrambling signatures.
} 
We notice that $d_{E \text {, min }}$ maximization results in the best performance only when the number of streams is low, although one could expect that, using a maximum-likelihood receiver, this would be the best approach for any number of streams. However, in the sub-optimal SIC receiver herein employed, the EMSD introduces cross-stream interference which depends on the number of streams. Such interference is only partially removed by the ETCMD decoders. We believe that design approaches other than $d_{E \text {, min }}$ maximization prove more efficient in mitigating such cross-stream interference and hence result in better performance.

\section{B. ETCMA Receiver}

A new receiver that performs significantly better than the original TCMA receiver [7] has been devised. As shown in Fig. 1(b) it consists of an Enhanced Multi-Stream Detector (EMSD) followed by a bank of single-stream Enhanced TCM Decoders (ETCMD) ${ }^{2}$. The receiver iteratively executes the EMSD and ETCMDs according to a Successive Interference Cancellation (SIC) schedule [13] which consists of two nested iteration loops. The outer iterations are indexed by variable $t=0, \ldots, N_{\text {IT }}-1$, where the number of outer iterations $N_{\text {IT }}$ is a receiver parameter. For each outer iteration $K$ inner iterations, indexed by variable $k=0, \ldots, K-1$, are executed.

In each inner iteration, as shown in Fig. 1(b), the EMSD calculates a vector of a-priori Log-Likelihood Ratios (LLRs) $\left\{\sigma_{k, n}^{(t)}(l)\right\}_{n=0}^{\left|\chi_{\mathrm{TCM}}\right|-1}$ for the $k$ th stream modulation symbols whose elements are computed ${ }^{3}$ as

$$
\begin{aligned}
\sigma_{k, n}^{(t)}(l) & =\log \frac{P\left(s_{k}(l)=m_{n} \mid \mathbf{r}\right)}{P\left(s_{k}(l)=m_{0} \mid \mathbf{r}\right)} \\
& =\underset{q: s_{k}\left(x_{q}(l)\right)=m_{n}}{\max _{k-1, q}^{*}}(l) \\
& -\underset{q: s_{k}\left(x_{q}(l)\right)=m_{0}}{\max _{k-1, q}^{*}} \Lambda_{k}^{(t)}(l)-\omega_{k, n}^{(t-1)}(l)
\end{aligned}
$$

where $m_{n}$ is the $n$th symbol of $\chi_{\mathrm{TCM}}\left(n=0, \ldots,\left|\chi_{\mathrm{TCM}}\right|-1\right)$, $x_{q}(l)$ is the $q$ th symbol of $\chi_{\mathrm{SUP}}(l)\left(q=0, \ldots,\left|\chi_{\mathrm{SUP}}\right|-1\right)$ and $\mathbf{r}=(r(0), \ldots, r(L-1))$ is the received vector. $\Lambda_{k-1, q}^{(t)}(l)$ (hereinafter called joint LLR distribution) represents the LLR of the $q$ th symbol of $\chi_{\mathrm{SUP}}(l)$ updated using the a-posteriori extrinsic LLR of the $n$-th TCM symbol $\omega_{k-1, n}^{(t)}$. Here and below we use the shorthand notation $\max ^{*}(a, b)$ to denote $\log \left(e^{a}+e^{b}\right)$.

LLRs $\sigma_{k, n}^{(t)}(l)$ are deinterleaved obtaining $\tau_{k, n}^{(t)}(l)=$ $\sigma_{k, n}^{(t)}\left(\Pi_{k}^{-1}(l)\right)$ and sent to $\mathrm{ETCMD}_{k}$ which executes the BCJR algorithm [15] on the trellis of the TCM encoder working at symbol level as in [16] and computes updated $a$ posteriori extrinsic LLRs $\nu_{k, n}^{(t)}(l)$. After interleaving, we obtain $\omega_{k, n}^{(t)}(l)=\nu_{k, n}^{(t)}\left(\Pi_{k}(l)\right)$.

Finally, the EMSD updates the joint LLR distribution as

$$
\Lambda_{k, q}^{(t)}(l)=\Lambda_{k-1, q}^{(t)}(l)+\omega_{k, n}^{(t)}(l)-\omega_{k, n}^{(t-1)}(l) .
$$

${ }^{2}$ We call our TCM decoder enhanced in order to remark that it is different from the original TCM decoder used in [7].

${ }^{3}$ See $[14]$ and references therein for details about probability computations in the LLR domain.

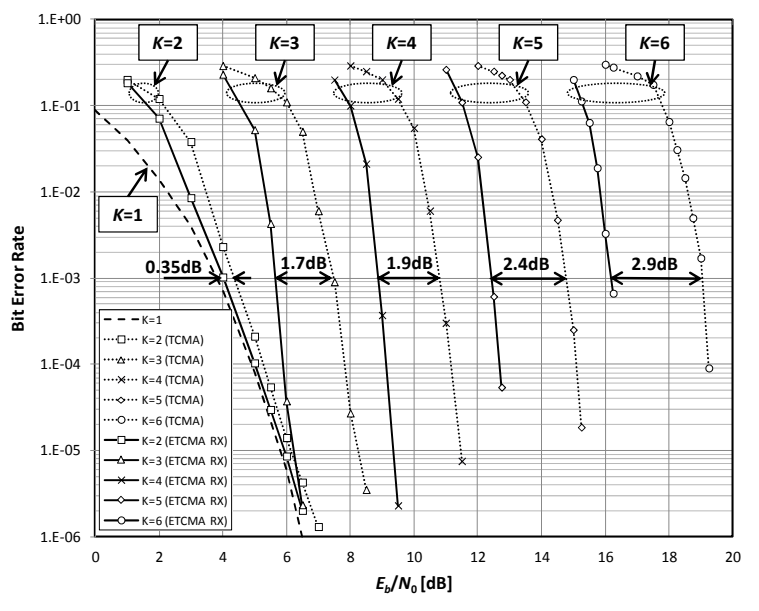

Fig. 2. Bit error rate of TCMA with enhanced receiver (solid curves) and original TCMA receiver [7] (dotted curves). Block length is $L=1000$ bits and the receiver performs $N_{\text {IT }}=5$ iterations.

Here and in 3 , when $k=0$ we set $\Lambda_{-1, q}^{(t)}(l)=\Lambda_{K-1, q}^{(t-1)}(l)$ for all $q, l$ and $t \geq 1$. When $k=0$ and $t=0$, we set

$$
\begin{aligned}
\Lambda_{-1, q}^{(0)}(l) & =\log \frac{P\left(s(l)=x_{q}(l) \mid \mathbf{r}\right)}{P\left(s(l)=x_{0}(l) \mid \mathbf{r}\right)} \\
& =\frac{\left\|r(l)-x_{0}(l)\right\|^{2}-\left\|r(l)-x_{q}(l)\right\|^{2}}{2 \sigma_{w}^{2}}
\end{aligned}
$$

where $x_{q}(l) \in \chi_{\mathrm{SUP}}(l)$ and $\sigma_{w}^{2}$ is the variance of noise. The LLRs $\omega_{k, n}^{(-1)}(l)$ are set to zero for all $k, n$ and $l$.

After a fixed number of iterations $N_{\mathrm{IT}}$, the receiver computes the a-posteriori LLRs of information bits $u_{k}(l)$ and delivers the decoded information $\hat{u}_{k}(l)$ to the recipient.

In the original TCMA receiver [7], the soft information values exchanged through the interleavers during the iterative process are LLRs of coded bits $\beta_{k}^{(i)}(l)=$ $\log \left[P\left(d_{k}^{(i)}(l)=1 \mid \mathbf{r}\right) / P\left(d_{k}^{(i)}(l)=0 \mid \mathbf{r}\right)\right]$. Computing the LLRs of TCM symbols - as in the ETCMA receiver - instead of coded bits - as in the original TCMA receiver - results in a slightly increased computational complexity and a significantly improved performance as shown in Fig. 2. where the Bit Error Rate (BER) of the ETCMA receiver is compared with the results of [7]. We obtain SNR gains that increase with the number of streams and reach $2.9 \mathrm{~dB}$ when $K=6$.

The overall receiver complexity is largely dominated by equations (3) and (4), whose number of operations grows exponentially with $K$ as the size of the super-constellation $\left|\chi_{\mathrm{SUP}}\right| \sim\left|\chi_{\mathrm{TCM}}\right|^{K}$. Simplified EMSD algorithms that compute (3) and (4) on suitably chosen subsets of $\chi_{\text {SUP }}$ yielding significant complexity reductions are being investigated.

\section{TCM Encoder Design}

The employed TCM scheme depends on the overloading factor $K$ and consist of a rate 1/2 convolutional encoder (CE) connected to a QPSK symbol mapper. We consider two CE schemes: a four-state CE with generator coefficients $(5,7)_{8}$ and a two-state CE with generator coefficients $(2,3)_{8}$ (see Fig. 3 ). 
Using EXtrinsic Information Transfer (EXIT) charts [17], [18], the behavior of the ETCMA receiver has been analyzed, yielding the conclusion that, when $K \leq 3$ four state encoders result in better performance whilst for $K \geq 4$ two-state encoders should be used. The EXIT charts, described in terms of average mutual information functions $I_{\sigma, k}^{(t)}, I_{\tau, k}^{(t)}, I_{\nu, k}^{(t)}$ and $I_{\omega, k}^{(t)}$, are shown in Fig. 4 Solid curves represent the ETCMD characteristics $\left(I_{\tau, k}\right.$ vs. $\left.I_{\nu, k}\right)$, while dashed curves represent the characteristics of the EMSD $\left(I_{\sigma, k+1}\right.$ vs. $\left.I_{\tau, k}\right)$, which depend both on the SNR and on $K$. Using an approach similar to [16], the average mutual information $I_{\omega, k}^{(t)}=\mathbb{E}_{l}\left[I\left(s_{k}(l) ; \omega_{k}^{(t)}(l)\right)\right]$ between the modulation symbols $s_{k}(l)$ and the LLRs $\omega_{k, n}^{(t)}(l)$ at iteration $t$ has been estimated as

$$
I_{\omega, k}^{(t)}=\log _{2}\left|\chi_{\mathrm{TCM}}\right|-\frac{1}{L} \sum_{l=0}^{L-1}\left(\max _{n}^{*}\left(\omega_{k, n}^{(t)}(l)\right)-\omega_{k, \bar{n}_{k, l}}^{(t)}(l)\right)
$$

where $\bar{n}_{k, l}$ is the index of the transmitted symbol: $s_{k}(l)=m_{\bar{n}_{k, l}}$. Similarly, we estimated $I_{\sigma, k}^{(t)}, I_{\tau, k}^{(t)}$ and $I_{\nu, k}^{(t)}$.

If an EMSD curve and an ETCMD curve intersect each other in a point close to the upper right corner - where $I_{\sigma}=I_{\omega}=2$ - then the decoder will converge to the correct code word and deliver an error-free information word. This is the case of the EMSD characteristic for $K=2$ streams at $\mathrm{SNR}=5 \mathrm{~dB}$ (upper red dashed curve of Fig. 4) and the four-state ETCMA characteristic (solid green curve in Fig. 4). Choosing the twostate TCM (solid blue curve in Fig. 4), the intersection would be moved to a point with lower $I_{\sigma}$ and $I_{\omega}$, thus predicting a higher error rate. The same considerations lead to the choice of the four-state encoder for $K=3$. When $K=4$, at SNR of $9 \mathrm{~dB}$ the EMSD characteristics intersects the four-state ETCMD characteristic at rather low values $I_{\sigma}$ and $I_{\omega}$, whilst the intersection with the two-state ETCMD characteristic is much closer to $I_{\sigma}=I_{\omega}=2$. A similar behavior is also expected when $K \geq 5$. As a result, when $K \geq 4$ we choose the two-state encoder.

\section{RESULTS}

Simulations have been performed in order to assess the performance of ETCMA schemes. The considered channel model is AWGN with two-sided power spectral density of noise $G_{w}(f)=N_{0} / 2$. The BLock Error Rate (BLER) has been evaluated and the aggregate SE has been computed as

$$
S E(K)=(1-\mathrm{BLER}) R m_{0} K \quad[\mathrm{bits} / \mathrm{s} / \mathrm{Hz}] .
$$

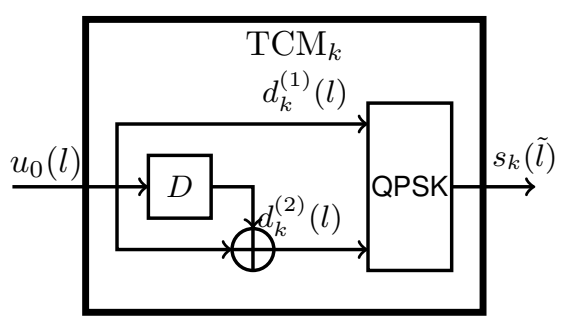

Fig. 3. TCM encoder-modulator. The two-state trellis encoder has generator coefficients $(2,3)_{8}$. Here, $\tilde{l}=\Pi_{k}^{-1}(l)$.

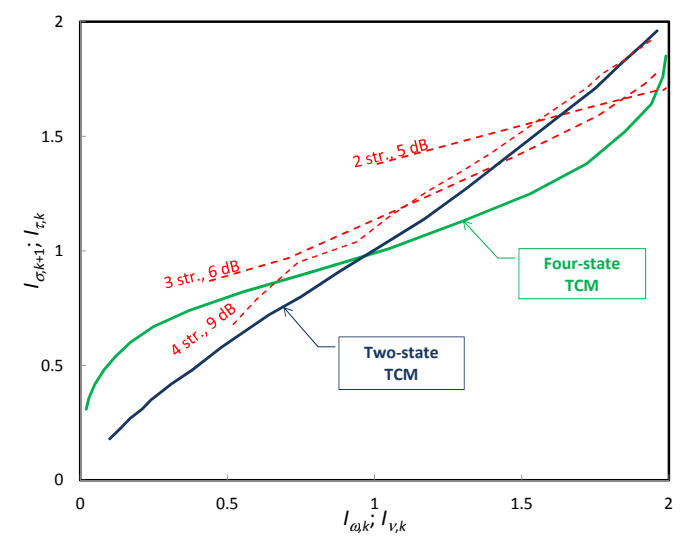

Fig. 4. EXIT charts of ETCMA receiver. Solid curves refer to the ETCMDs; dashed curves refer to the EMSD.

TABLE I

TRANSMISSION PARAMETERS.

\begin{tabular}{cccc}
$K$ & $\begin{array}{c}\text { TCM constraint } \\
\text { length }\end{array}$ & $\begin{array}{c}\text { TCM } \\
\text { modulation }\end{array}$ & $\begin{array}{c}\text { Scrambling } \\
\text { sequence type }\end{array}$ \\
\hline 2 & 3 & QPSK & Max. $d_{E, \text { min }}$ \\
3 & 3 & QPSK & Uniformly spaced \\
4,5 & 2 & QPSK & Uniformly spaced \\
6,7 & 2 & QPSK & Zadoff-Chu
\end{tabular}

Here, $R=1 / 2$ is the channel code rate and $m_{0}=2$ is the modulation order. The asymptotic aggregate SE (ASE) is

$$
S E_{\infty}(K)=\lim _{\mathrm{SNR} \rightarrow \infty} S E(K) .
$$

The most relevant metric we take into account is the singlestream SNR loss $\Delta_{\mathrm{SNR}}(K, \rho)$, which is the increase of SNR with respect to the single-stream SNR required to achieve a given ratio $\rho$ of the ASE when the overloading factor is $K>1$ :

$$
\begin{aligned}
\Delta_{\mathrm{SNR}}(K, \rho) & =\left.\mathrm{SNR}\right|_{S E(K)=\rho S E_{\infty}(K)} \\
& -\left.\mathrm{SNR}\right|_{S E(1)=\rho S E_{\infty}(1)} .
\end{aligned}
$$

Each information word is transmitted using $L=240$ REs. The transmission parameters are selected according to Tab. I

The receiver performs $N_{\text {IT }}$ outer iterations. Decoding complexity has been taken into account for the determination of the number of iterations. When the four-state TCM encoder is used, the receiver executes $N_{\text {IT }}=10$ iterations. Using two-state encoders, the trellis complexity of TCM is reduced, therefore we increase the number of iterations to $N_{\text {IT }}=15$ in order to partially compensate for the reduced complexity of ETCMDs. Fig. 5 shows the SE of ETCMA on the AWGN channel. In Fig. 5(a) the SE of ETCMA is compared with the SE of the original TCMA [7]. The SNR loss of ETCMA computed applying (7) is summarized in Tab. II and therein compared with the SNR loss fo TCMA. The observed SNR gains ${ }^{4}$ increase with $K$ and range from less than $0.35 \mathrm{~dB}$ for $K=2$ to a remarkable $7.15 \mathrm{~dB}$ for $K=7$. The AWGN channel capacity is also shown as a reference. We observe that

\footnotetext{
${ }^{4}$ The SNR gains here reported are different from those shown in Fig. 2 because of the different number of receiver iterations.
} 


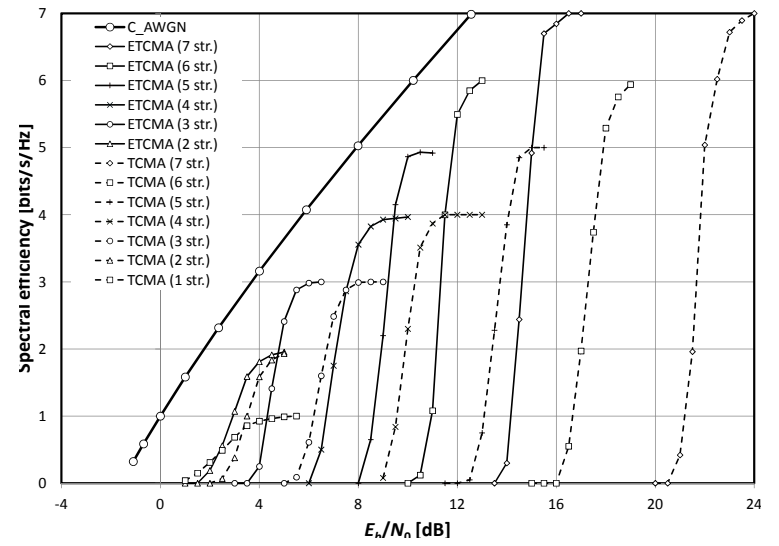

(a) Aggregate SE of ETCMA (solid curves) compared with the SE obtained using the original TCMA receiver [7] (dashed curves).

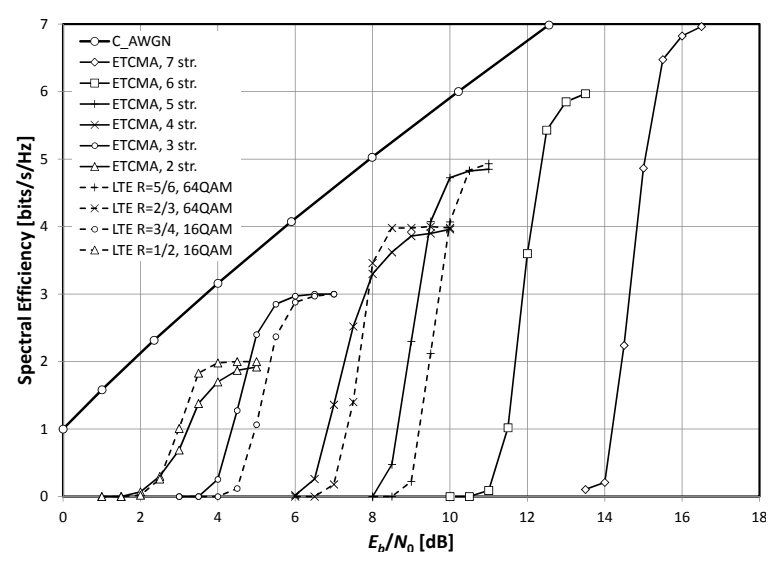

(b) SE of ETCMA (solid curves) compared with turbo coded LTE (dashed curves).

Fig. 5. Spectral efficiency of ETCMA.

TABLE II

SingLE-STREAM SNR LOSSES $\Delta_{\text {SNR }}(K, \rho)$ OF TCMA AND ETCMA FOR $\rho=0.9$. RIGHTMOST COLUMN: SNR GAIN OF ETCMA OVER TCMA

\begin{tabular}{cccc}
$K$ & TCMA [dB] & ETCMA [dB] & SNR gain [dB] \\
\hline 2 & 0.6 & 0.25 & 0.35 \\
3 & 3.4 & 1.55 & 1.85 \\
4 & 6.75 & 4.3 & 2.45 \\
5 & 10.45 & 6.0 & 4.45 \\
6 & 14.25 & 8.2 & 6.05 \\
7 & 18.8 & 11.65 & 7.15
\end{tabular}

ETCMA exhibits a much smaller SNR gap of about $2 \div 2.5$ dB w.r.t. the AWGN capacity.

Fig. 5(b) compares the SE of ETCMA with the SE of a turbo coded LTE link [19]. Both systems use $L=240$ REs per data block. In the LTE system, an information word of $K L$ bits is transmitted using $L$ REs. The ETCMA transmitter segments the information word into $K$ sub-words of length $L$ which are independently encoded and combined then transmitted. At the receiver, the inverse procedure is performed in order to reassemble the information word. SE is evaluated using (5) but here, as a difference w.r.t. Fig. 5(a), BLER is computed over blocks of size $K L$ bits. ETCMA exhibits a higher SE than LTE for several SNR values and achieves SE of $6 \mathrm{bits} / \mathrm{s} / \mathrm{Hz}$ and above that are not achievable with current LTE systems, while performing close to the AWGN channel capacity.

\section{CONCLUSIONS}

We have proposed a new OLMA scheme that, compared to original TCMA, provides large SNR gains of up to 7.15 $\mathrm{dB}$ at $7 \mathrm{bits} / \mathrm{s} / \mathrm{Hz}$. In terms of aggregate SE, it approaches the capacity of the AWGN channel within a SNR gap of about 2 $\mathrm{dB}$ over a wide range of spectral efficiencies.

\section{REFERENCES}

[1] A. Kapur and M. Varanasi, "Multiuser detection for overloaded CDMA systems," IEEE Trans. Inf. Theory, vol.49, no.7, pp. 1728-1742, Jul 2003.

[2] J. Choi, "Low density spreading for multicarrier systems," in IEEE 8th Int. Symp. Spread Spectrum Techniques and Applications, 2004, Aug 2004, pp. 575-578.
[3] J. van de Beek and B. M. Popović, "Multiple access with low-density signatures," in IEEE Global Telecommun. Conf., GLOBECOM 2009, Nov 2009, pp. 1-6.

[4] B. M. Popović, A. R. Safavi, and A. G. Perotti, "Bit-interleaved low density spread (BI-LDS) transmission," in IEEE Wireless Commun. Networking Conf., WCNC 2014, Apr 2014.

[5] L. Ping et al., "Interleave division multiple-access," IEEE Trans. Wireless Commun., vol. 5, no. 4, pp. 938-947, April 2006.

[6] T. Aulin and R. Espineira, "Trellis coded multiple access (TCMA)," in IEEE Int. Conf. Commun., ICC 1999, vol. 2, 1999, pp. 1177-1181 vol.2.

[7] F. Brännström et al., "Iterative detectors for trellis-code multiple-access," IEEE Trans. Commun., vol. 50, no. 9, pp. 1478-1485, Sep 2002.

[8] G. Caire, G. Taricco, and E. Biglieri, "Bit-interleaved coded modulation," IEEE Trans. Inf. Theory, vol. 44, pp. 927-946, May 1998.

[9] G. Ungerboeck, "Channel coding with multilevel/phase signals," IEEE Trans. Inf. Theory, vol. 28, no. 1, pp. 55-67, Jan 1982.

[10] J. Sun and O. Takeshita, "Interleavers for turbo codes using permutation polynomials over integer rings," IEEE Trans. Inf. Theory, vol. 51, no. 1 , pp. 101-119, Jan 2005.

[11] F. N. Brännström, T. M. Aulin, and L. K. Rasmussen, "Constellationconstrained capacity for trellis code multiple access systems," in IEEE Global Telecommun. Conf., GLOBECOM '01, vol. 2, 2001, pp. 791-795.

[12] B. M. Popović, "Generalized chirp-like polyphase sequences with optimum correlation properties," IEEE Trans. Inf. Theory, vol. 38, no. 4, pp. 1406-1409, Jul 1992.

[13] D. Tse and P. Viswanath, Fundamentals of wireless communications. Cambridge University Press, 2005.

[14] S. Benedetto et al., "Soft-input soft-output modules for the construction and distributed iterative decoding of code networks," Eur. Trans. Telecomm., vol. 9, pp. 155-172, March 1998.

[15] L. R. Bahl et al., "Optimal decoding of linear codes for minimizing symbol error rate," IEEE Trans. Inf. Theory, vol. 20, pp. 284-287, March 1974.

[16] B. Scanavino, G. Montorsi, and S. Benedetto, "Convergence properties of iterative decoders working at bit and symbol level," in IEEE Global Telecommun. Conf., GLOBECOM '01, vol. 2, 2001, pp. 1037-1041.

[17] S. ten Brink, "Convergence behavior of iteratively decoded parallel concatenated codes," IEEE Trans. Commun., vol. 49, no. 10, pp. 17271737 , Oct 2001

[18] F. Brännström et al., "Convergence analysis of iterative detectors for narrow-band multiple access," in IEEE Global Telecommun. Conf., GLOBECOM '02., vol. 2, Nov 2002, pp. 1373-1377.

[19] "Third Generation Partnership Project; Technical Specification Group Radio Access Network; Evolved Universal Terrestrial Radio Access (E-UTRA); Multiplexing and channel coding (Release 12)," 3GPP TS 36.212 V12.0.0, Dec 2013 